\title{
miR-217 regulates tumor growth and apoptosis by targeting the MAPK signaling pathway in colorectal cancer
}

\author{
NAN ZHANG, CANRONG LU and LIN CHEN \\ General Surgery Center Department of Chinese PLA General Hospital, Beijing 100853, P.R. China
}

Received May 16, 2015; Accepted September 28, 2016

DOI: $10.3892 / \mathrm{ol} .2016 .5249$

\begin{abstract}
MicroRNA (miR)-217 has been reported to participate in carcinogenesis and tumor progression in several cancers; however, its expression and biological functions in colorectal cancer (CRC) are still unclear. The present study demonstrated that miR-217 expression was significantly higher in matched adjacent noncancerous tissues than in CRC tissues $(\mathrm{P}<0.001)$. In addition, it was observed that low-grade CRC exhibited greater expression of miR-217 compared with high-grade CRC $(\mathrm{P}<0.05)$. Kaplan-Meier survival and Cox regression analyses revealed that overall survival rates were significantly poorer in the low-expression group relative to the high-expression group $(\mathrm{P}<0.005)$. Next, a potential miR-217 target, mitogen-activated protein kinase (MAPK) 1, was identified. Upregulation of miR-217 could significantly downregulate MAPK1 expression. CRC cells overexpressing miR-217 exhibited cell growth inhibition by significant enhancement of apoptosis in vitro. The present study further investigated the MAPK signaling pathway to verify the mechanisms, and revealed that KRAS and Raf-1 expression was downregulated in miR-217-overexpressing RKO cells. Taken together, our results revealed that miR-217 inhibits tumor growth and enhances apoptosis in CRC, and that this is associated with the downregulation of MAPK signaling. These results indicate that miR-217 is a promising therapeutic target for the treatment of CRC.
\end{abstract}

\section{Introduction}

Colorectal cancer (CRC) represents the third most common cancer in men and the second most common cancer in women globally (1-3). With the development of early diagnosis and treatment modalities, the 5-year survival rate of CRC has been improved over the past two decades $(2,4)$. Currently, combination chemotherapy strategy has been recognized as the standard of care in international guidelines $(4,5)$. However,

Correspondence to: $\mathrm{Dr}$ Lin Chen, General Surgery Center Department of Chinese PLA General Hospital, 28 Fuxing Road, Haidian, Beijing 100853, P.R. China

E-mail: chenlin301@sina.vip.com

Key words: miR-217, colorectal cancer, apoptosis, MAPK1 even with aggressive intervention, it does not ensure a cure, particularly for patients with malignant CRC $(6,7)$. Research has been focused on tumor-suppressor genes, oncogenes and cell signaling pathways, including their role in the proliferation, apoptosis and aggressiveness of these tumors (8). The resulting information has led the way for an increasing interest in potential genetics-based treatments (9).

MicroRNAs (miRNAs or miRs) are a class of noncoding RNA molecules that negatively regulate the translation of messenger (m) RNAs by interacting with complementary sites in the 3' untranslated region (UTR) (10). miRNAs regulate various biological processes, including cell growth, invasion, cell cycle and apoptosis (10). The close association between miRNAs and tumors is also well studied (11). Several miRNAs have been regarded as oncomiRs or tumor suppressor genes in specific cancers by directly targeting oncogenes or tumor suppressor genes (12). Recently, mounting evidence indicates that miR-217 can function as a tumor suppressor gene or as an oncogene depending on the cell type $(13,14)$. miR-217 has been proved to target the oncogenes sirtuin 1 (SirT1) or KRAS in endothelial and pancreatic ductal adenocarcinoma cells, although it targets the tumor suppressor gene phosphatase and tensin homolog (PTEN) in kidney (13-16). However, its function and expression in CRC are still unclear.

The present study identified that mitogen-activated protein kinase (MAPK) 1 is a novel miR-217 target, which is regarded as a critical component of the MAPK signaling pathway (17). Considering that the MAPK signaling pathway is important in regulating pathological cell growth (18), it is considered that downregulation of MAPK inhibitors would represent an ideal anti-tumor therapy. It was additionally demonstrated that miR-217 could inhibit tumor proliferation and enhance apoptosis of CRC via the MAPK pathway.

\section{Materials and methods}

Cell culture. The malignant CRC cell lines RKO and SW480 were obtained from the Chinese Academy of Sciences (Shanghai, China). All cells were cultured in Dulbecco's modified Eagle medium (Lonza Inc., Allendale, NJ, USA) supplemented with $0.1 \%$ insulin, $50 \mathrm{U} / \mathrm{ml}$ penicillin/streptomycin, $1 \%$ non-essential amino acids and $10 \%$ fetal bovine serum (Lonza Inc.) at $37^{\circ} \mathrm{C}$ in an atmosphere of $5 \% \mathrm{CO}_{2}$. All cell lines were tested periodically for mycoplasma contamination using MycoAlert Mycoplasma Detection kit (Lonza Inc.). 
Transfection and luciferase activity assay. miRNA transfection and luciferase activity assay were performed as described previously (13). Briefly, the human CRC cell lines RKO and SW480 were seeded on 24-well plates and transfected $24 \mathrm{~h}$ later using Lipofectamine 2000 according to the manufacturer's protocol (Invitrogen; Thermo Fisher Scientific, Inc., Waltham, MA, USA). The miR-217 precursor molecules (Ambion; Thermo Fisher Scientific, Inc.) were co-transfected with $4 \mathrm{ng} /$ well of plasmid pRL-CMV (Promega Corporation, Madison, WI, USA) and $80 \mathrm{ng} /$ well of luciferase reporter plasmids (Shanghai GenePharma Co., Ltd., Shanghai, China).

Tissue samples. The present study protocol complies with National Regulations on the Use of Clinical Samples in China (19), and was approved by the by Ethics Committee of Chinese PLA General Hospital (Beijing, China). CRC specimens were obtained from patients with CRC at the Chinese PLA General Hospital from July 2000 to July 2006. Follow-up was conducted in all patients, and survival time was completed in December 2009. The follow-up was carried out every 3 months by e-mail or telephone, and the last follow-up was in January 2010. The clinicopathological parameters of the patients are shown in Table I. Written informed consent was obtained from all patients.

Protein extraction and western blotting. Protein extraction and western blot analysis were conducted as described previously (20). The primary antibodies used were anti-MAPK1 (Abcam, Cambridge, UK, ab102930, 1:500), anti-p38 MAPK (Abcam, ab7952, 1:800), anti-B-cell lymphoma (Bcl)-2 (Sigma-Aldrich; Merck Millipore, Darmstadt, Germany, SAB4300339, 1:500), anti-Raf-1 (Abcam, ab32025, 1:800) and anti- $\beta$-actin (Santa Cruz Biotechnology, Inc., sc-8432, 1:500).

Reverse transcription-quantitative polymerase chain reaction analysis (RT-qPCR). The RNeasy Mini kit (Qiagen $\mathrm{GmbH}$, Hilden, Germany) was used to extract total RNA from RKO cells and frozen tissue specimens. The expression of miR-217 was measured using the standard TaqMan ${ }^{\circledR}$ MicroRNA Assay (Applied Biosystems; Thermo Fisher Scientific, Inc., Waltham, MA, USA) as previously reported (21). The primers were designed as follows: MAPK1, 5'-ATG ACCTCCTATGGCATCGA-3' (forward) and 5'-TGATGT TCTGTGCGTGGAGC-3' (reverse); Bcl-2, 5'-CCGGGAGAT CGTGATGAAGT-3' (forward) and 5'-ATCCCAGCCTCC GTTATCCT-3' (reverse); Bcl-extra large (xl), 5'-ATGGAG AACAATAAAACCT-3' (forward) and 5'-CTAGTGATA AAAGTAGAGTTC-3' (reverse); and KRAS, 5'-GGCCTG CTGAAAATGACTGAAT-3' (forward) and 5'-ATTGTT GGATCATATTCGTCCAC-3' (reverse). First-strand complementary DNA was synthesized using a ReverTra Ace ${ }^{\circledR}$ qPCR RT kit (Toyobo, Co., Ltd., Osaka, Japan). RT-qPCR was performed using the ABI PRISM ${ }^{\circledR} 7900$ HT Sequence Detection System (Applied Biosystems; Thermo Fisher Scientific, Inc.) under the following cycling conditions: $95^{\circ} \mathrm{C}$ for $3 \mathrm{~min}$ followed by 40 cycles of $95^{\circ} \mathrm{C}$ for $10 \mathrm{sec}$ and $55^{\circ} \mathrm{C}$ for $45 \mathrm{sec}$. Melting curve analysis was used to check the specificity of amplification. Relative gene expression was calculated by using the comparative $\mathrm{Cq}$ method (22).
Apoptosis assay. An Annexin V-fluorescein isothiocyanate (FITC) apoptosis kit was used to measure apoptosis according to the manufacturer's instructions (ImmunoChemistry Technologies, LLC, Bloomington, MN, USA). Cells were analyzed by flow cytometry after being stained with an anti-Annexin V-FITC antibody (Santa Cruz Biotechnology, Inc., sc-1929, 1:1,000). For each experiment, at least 20,000 cells were analyzed.

Colony formation and proliferation assay. Colony forming capacity was evaluated as previously described (23). At daily intervals, the number of viable cells was determined by MTT assay to measure the cell proliferation. Cell proliferation was analyzed by Cell Counting kit (Sigma-Aldrich; Merck Millipore, 03285) according to the manufacturer's instructions. The optical density was measured by using a microplate reader (Thermo Fisher Scientific, Inc.) at $570 \mathrm{~nm}$.

Statistics. The $\chi^{2}$ test was used to analyze the miR-217 expression and clinicopathological characteristics. Cox proportional-hazards regression analysis was used to evaluate predictors of survival. Overall survival times in CRC patients were compared using Kaplan-Meier survival analysis. Analyses were performed using SPSS 10.0 (SPSS, Inc., Chicago, IL, USA). $\mathrm{P}<0.05$ was considered to indicate a statistically significant difference.

\section{Results}

miR-217 expression and association with clinicopathological characteristics in $C R C$. The expression levels of miR-217 were measured in 121 human CRC specimens and 48 matched adjacent noncancerous tissues. As shown in Fig. 1A, the expression levels of miR-217 were markedly higher in normal tissues compared with those in CRC tissues (mean \pm standard deviation: $7.82 \pm 1.8$ vs. $2.71 \pm 0.89, \mathrm{P}<0.001$ ). In addition, it was observed that low-grade CRC [World Health Organization (WHO) grade I, 3.12 \pm 1.08 ] exhibited greater expression of miR-217 compared with high-grade CRC (WHO grade II-III, 1.98 $\pm 1.16, \mathrm{P}<0.05$ ). Thus, miR-217 expression is associated with the proliferation of CRC and with tumor progression.

To explore whether miR-217 expression is correlated with clinicopathological parameters, the association between miR-217 expression and clinical characteristics of patients with CRC was explored. The mean miR-217 expression level of CRC was 2.71, which was utilized to divide high-grade CRC patients into a high group $(\geq 2.71, n=68)$ and a low group $(<2.71, \mathrm{n}=53)$. Univariate analysis indicated a significant association between miR-217 expression and prognosis (Table II), while no significant associations were identified between prognosis and other clinical characteristics. The Kaplan-Meier survival curves revealed that the survival rate probability was higher in CRC patients exhibiting high miR-217 expression than in patients with low miR-217 expression (Fig. 1B). Multivariate analysis further revealed that miR-217 expression is an independent factor for overall survival (Table II). These data suggest that miR-217 expression may be a critical prognostic factor for high-grade CRC. 
Table I. Association between miR-217 expression and clinicopathological variables of colorectal cancer patients.

\begin{tabular}{|c|c|c|c|c|}
\hline \multirow[b]{2}{*}{ Characteristics } & \multirow[b]{2}{*}{ No. of patients } & \multicolumn{2}{|c|}{ miR-217 expression } & \multirow[b]{2}{*}{$\underline{P \text {-value }}$} \\
\hline & & Low & High & \\
\hline No. of patients & 121 & 53 & 68 & \\
\hline Age, years & & & & 0.14 \\
\hline$<50$ & 71 & 32 & 39 & \\
\hline$\geq 50$ & 50 & 21 & 29 & \\
\hline Gender & & & & 0.10 \\
\hline Male & 69 & 28 & 41 & \\
\hline Female & 52 & 25 & 27 & \\
\hline $\mathrm{MTD}, \mathrm{cm}$ & & & & 0.08 \\
\hline$<5$ & 80 & 38 & 42 & \\
\hline$\geq 5$ & 41 & 15 & 26 & \\
\hline Tumor location & & & & 0.11 \\
\hline Rectum & 57 & 27 & 30 & \\
\hline Colon & 64 & 26 & 38 & \\
\hline Depth of invasion & & & & 0.14 \\
\hline $\mathrm{T} 1 / \mathrm{T} 2$ & 38 & 18 & 20 & \\
\hline $\mathrm{T} 3 / \mathrm{T} 4$ & 83 & 35 & 48 & \\
\hline Survival status & & & & 0.01 \\
\hline Alive & 23 & 5 & 18 & \\
\hline Dead & 98 & 48 & 50 & \\
\hline TNM stage & & & & 0.09 \\
\hline II & 49 & 24 & 25 & \\
\hline III/IV & 72 & 29 & 43 & \\
\hline
\end{tabular}

TNM, T category describes the primary tumor site; $\mathrm{N}$ category describes the regional lymph node involvement; $\mathrm{M}$ category describes the presence or otherwise of distant metastatic spread; MTD, mean tumor diameter; miR, microRNA.

MAPK1 is a potential target of miR-217. We hypothesized that MAPK1 is a potential target of miR-217 because it has three putative miR-217 target sites in its 3' UTR by using TargetScan (www.targetscan.org) (Fig. 1C). Luciferase reporter vectors that are fully complementary to either the full-length MAPK1 3' UTR or to the intact mature miR-7 sequence were used (Fig. 1D). Next, the mutant sequence of the putative target site or its relevant target site were cloned into an identical reporter vector to determine the major targets in RKO malignant CRC cells. By using a luciferase reporter assay, it was indicated that only when miR-217 was present, wild-type MAPK1 3' UTR and miR-217 could significantly reduce the relative luciferase activity (Fig. 1E and F). Furthermore, it was revealed that the reporter vectors with putative mir-217 target sites $\mathrm{A}$ or $\mathrm{C}$ resulted in $38.2 \pm 4.5 \%$ or $42.8 \pm 3.9 \%$ decrease in relative luciferase activity, respectively, compared with the corresponding mutant introduced with miR-217 in RKO cells (Fig. 1G). In addition, the relative luciferase activity was only reduced to $91.2 \pm 7.2 \%$ when the vector harbored only the putative target site B. Finally, it was observed that the relative luciferase activity was decreased to $19.1 \pm 1.4 \%$ by a new artificial target $\mathrm{D}$ (including putative target sites A and C) (Fig. 1G). This result was similar to the wild-type MAPK1 3' UTR-induced relative luciferase activity in RKO cells. Transfection of RKO cells with miR-217 resulted in a $\sim 5.6$-fold increase in miR-217 expression compared with mock- and miR-negative control (NC)-transfected cells, while MAPK1 mRNA was reduced to $24.8 \%$ when the RKO CRC cells were transfected with miR-217 (Fig. 1H). Western blotting revealed that miR-217 specifically decreased MAPK1 expression at the protein level (Fig. 1I) without affecting the expression of the other MAPK family members (p38 MAPK and extracellular-signalregulated kinase 5). Thus, these data suggest that MAPK1 is a novel target of miR-217.

miR-217 represses the survival of CRC cells in vitro. In light of the above findings, we tested the hypothesis that miR-217 may repress CRC cell proliferation and enhance cell apoptosis by inhibiting MAPK1 expression. To verify our hypothesis, an RKOcell line stably transfected with miR-217, miR-NC or mock (Null) was established to investigate the effects of miR-217 expression on CRC cell proliferation. It was observed that overexpression of miR-217 significantly inhibited tumor cell proliferation in stable miR-217-transfected RKO and SW480 cells compared with mock cells and miR-NC-transfected cells, as measured by daily MTT assays (Fig. 2A). Furthermore, colony formation assays revealed that transfection of RKO 


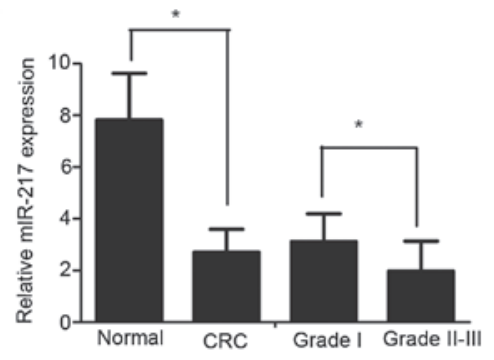

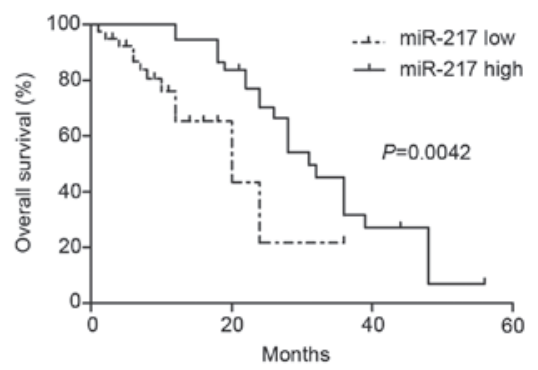

C

MAPK1 MRNA 3'UTR NM_002745



D



$\mathbf{F}$
E

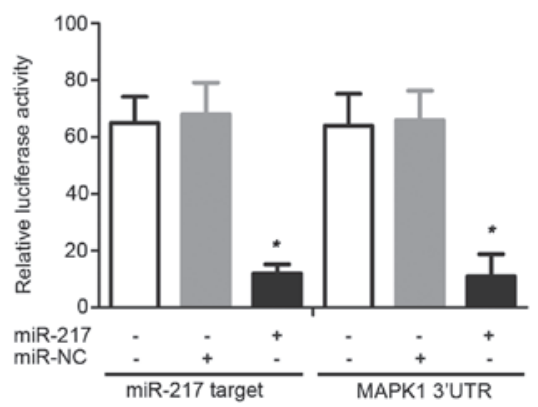

G

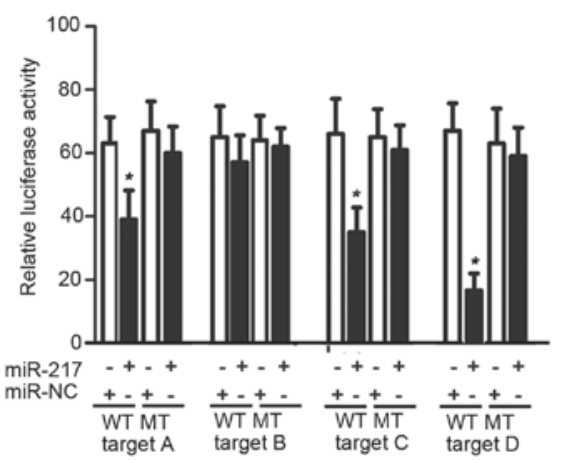

I

Figure 1. (A) miR-217 levels of were measured in CRC specimens by RT-qPCR. "P<0.05. (B) Kaplan-Meier survival curves according to miR-217 expression. ${ }^{*} \mathrm{P}=0.0042$. (C) Chart of MAPK1 mRNA sequence. Predicted consequential pairing of MAPK1 region: Region A, positions 395-401 of MAPK1 3' UTR; region B: positions 665-671 of MAPK1 3' UTR; region C, positions 778-784 of MAPK1 3' UTR. (D) Schematic diagram of luciferase reporter constructs transfected into RKO cells. (E) Luciferase reporter plasmids were co-transfected into RKO cells, and the relative luciferase activity was determined. "P<0.05. (F) Sequence of wild type and mutant miR-217 target sites in the MAPK1 3' UTR. (G) The contribution of the three miR-217 target sites was measured by a luciferase reporter assay ${ }^{*} \mathrm{P}<0.01$. (H) The expression level of miR-217 was measured in stably expressing subclones by TaqMan ${ }^{\circledR}$ MicroRNA Assay. RT-qPCR demonstrated that MAPK1 mRNA was significantly decreased in RKO cells transfected with miR-217 compared with mock- and miR-NC-transfected cells. ${ }^{*} \mathrm{P}<0.05$. (I) Western blot analysis revealed that miR-217 specifically repressed MAPK1 protein expression without influencing the expression of the other MAP kinase family members (p38 MAPK and ERK5). miR, microRNA; CRC, colorectal cancer; MAPK, mitogen-activated protein kinase; mRNA, messenger RNA; UTR, untranslated region; NC, negative control; hsa, Homo sapiens; WT, wild type; MT, mutant; ERK, extracellular signal-regulated kinase; RT-qPCR, reverse transcription-quantitative polymerase chain reaction.

and SW480 cells with miR-217 resulted in significantly lower anchorage-independent growth compared with cells transfected with miR-NC (Fig. 2B).
miR-217 enhances the apoptosis of CRC cells in vitro. Given the effects of miR-217 expression in RKO and SW480 cell proliferation, we hypothesized that miR-217 could regulate 
Table II. Univariate and multivariate analyses of different prognostic parameters in patients with colorectal cancer by log-rank test and Cox regression analysis.

\begin{tabular}{lccc}
\hline Variable & Univariate log-rank test (P-value) & Cox multivariate analysis (P-value) & Relative risk \\
\hline Age & 0.178 & 0.148 & 0.987 \\
Gender & 0.238 & 0.435 & 1.012 \\
MTD & 0.876 & 0.706 & 0.824 \\
Tumor location & 0.762 & 0.604 & 0.987 \\
Depth of invasion & 0.541 & 0.442 & 1.112 \\
TNM stage & 0.089 & 0.168 & 1.862 \\
miR-217 expression & 0.009 & 0.018 & 6.438
\end{tabular}

TNM, T category describes the primary tumor site; $\mathrm{N}$ category describes the regional lymph node involvement; $\mathrm{M}$ category describes the presence or otherwise of distant metastatic spread; MTD, mean tumor diameter; miR, microRNA.

A

RKO

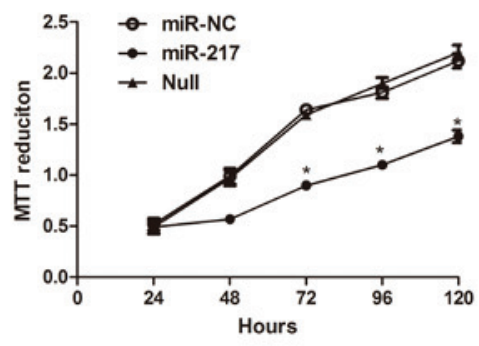

B

RKO
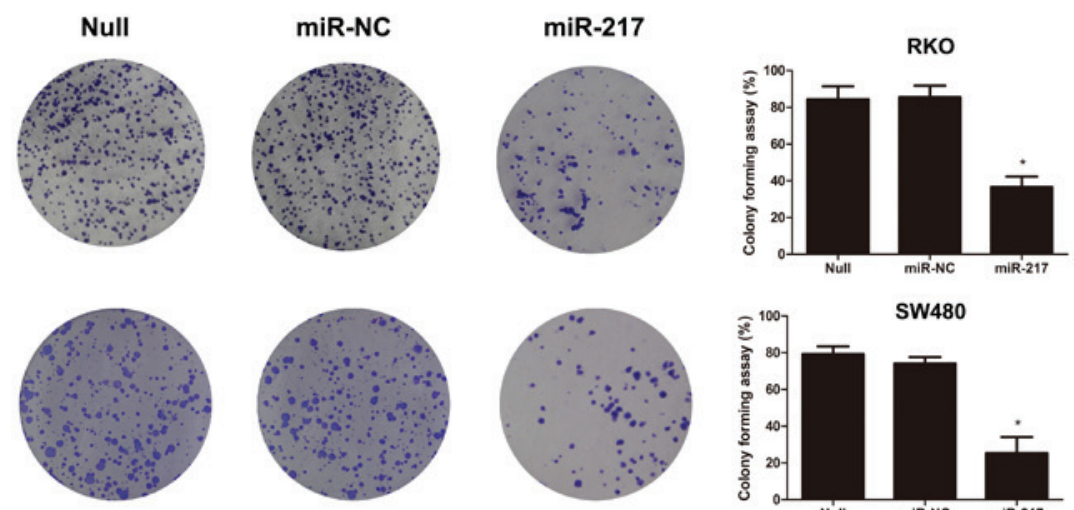

SW480

C

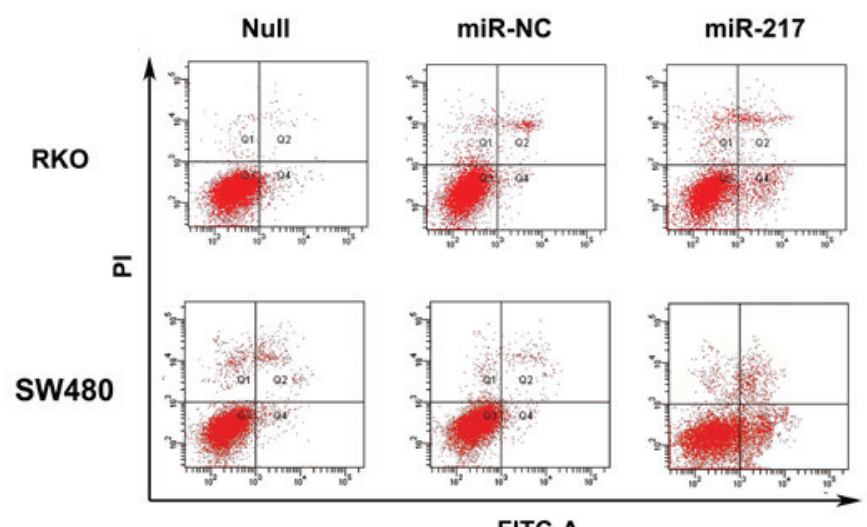

SW480

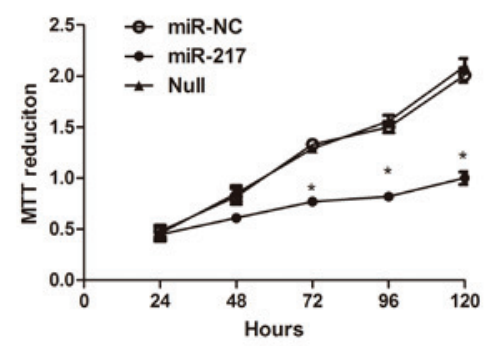

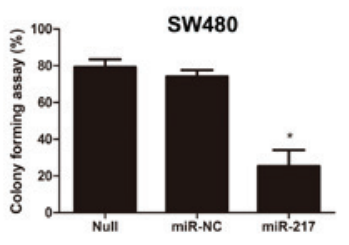
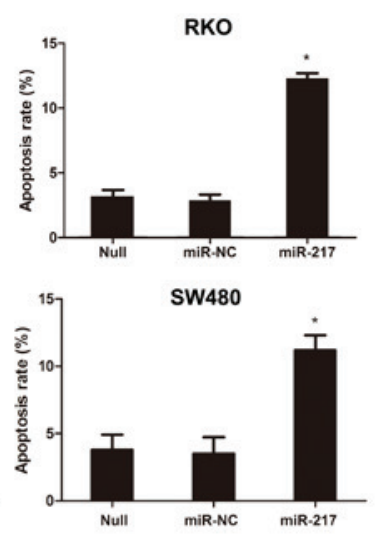

Figure 2. (A) Effect of miR-217 on RKO cell proliferation * $\mathrm{P}<0.05$. (B) Effect of miR-217 on RKO cell anchorage-dependent growth. "P<0.05. (C) The number of apoptotic cells was significantly higher in RKO cells transfected with miR-217, as measured by PI staining and flow cytometry ("P<0.05 for miR-217 vs. mock or miR-NC cells). miR, microRNA; NC, negative control; Q, quadrant; FITC-A, fluorescein isothiocyanate-Annexin V; PI, propidium iodide. 
A
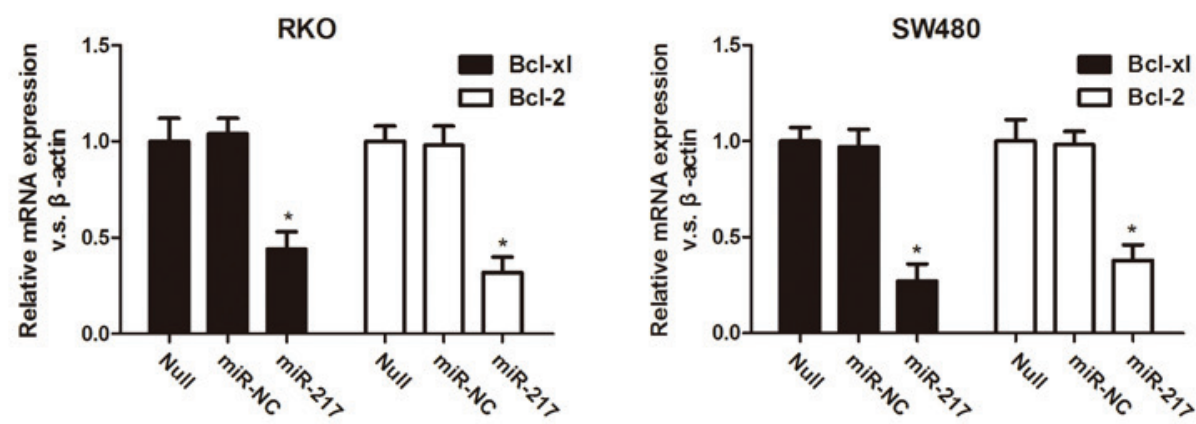

B

RKO


C

RKO

D
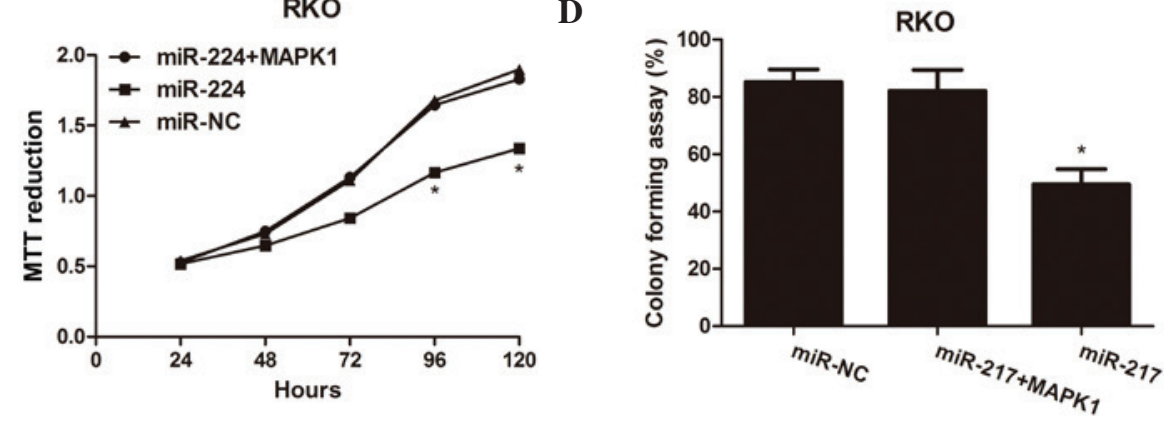

$\mathbf{E}$

$\mathbf{F}$


Figure 3. (A) Bcl-2 and Bcl-xl expression was measured by reverse transcription-quantitative polymerase chain reaction. " $\mathrm{P}<0.05$. (B) Western blot analysis revealed similar results. (C) RKO cells were co-transfected with miR-217 and MAPK1 overexpression plasmid or with a control, and the absorbance (representative of MTT reduction) was measured at 1, 2, 3, 4 or 5 days after transfection. "P<0.05 compared with the control groups. (D) Representative results of colony formation assay in RKO cells co-transfected with miR-217 precursor and MAPK1. " $\mathrm{P}<0.05$ compared with the control groups. (E) Apoptosis assay of RKO cells transfected with miR-217 precursor and MAPK1 overexpression plasmid or with a control. "P<0.05 compared with the control groups. (F) MAPK1, Bcl-2 and Bcl-xl protein expression was measured in RKO cells co-transfected with miR-217 precursor and MAPK1 overexpression plasmid or with a control. mRNA, messenger RNA; NC, negative control; miR, microRNA; Bcl, B-cell lymphoma; xl, extra large; MAPK, mitogen-activated protein kinase.

the apoptosis rate of CRC cells. As predicted, apoptosis assay indicated that RKO and SW480 cells stably transfected with miR-217 exhibited a higher apoptosis rate compared with RKO cells transfected with miR-NC (Fig. 2C). These results indicate that miR-217 may play a critical role in regulating cell apoptosis.

To determine the molecular mechanism of cell death induced by miR-217, the expression of Bcl-2 and Bcl-xl in these CRC transfectants was initially measured via RT-qPCR and western blotting. Treatment of RKO and SW480 cells with miR-217 significantly decreased Bcl-xl expression, which was associated with a reduction in activated Bcl-2 expression (Fig. 3A). Western blot analysis revealed similar results (Fig. 3B). Taken together, our results indicate that miR-217 is associated with apoptosis 
A

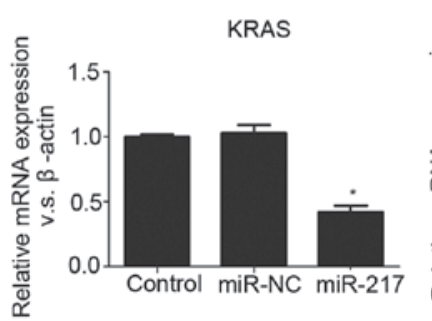

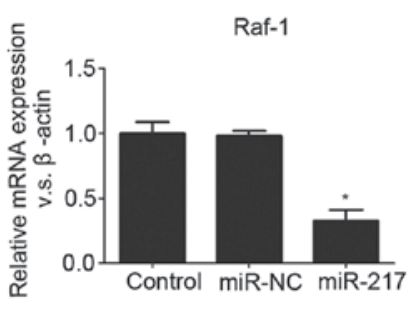

B

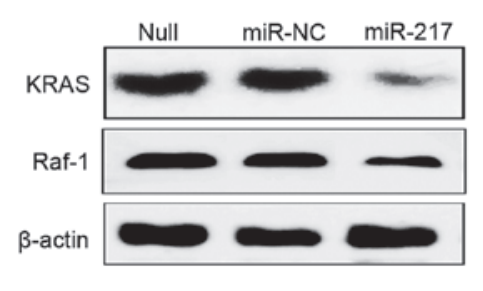

C

KRAS MRNA 3'UTR NM_033360

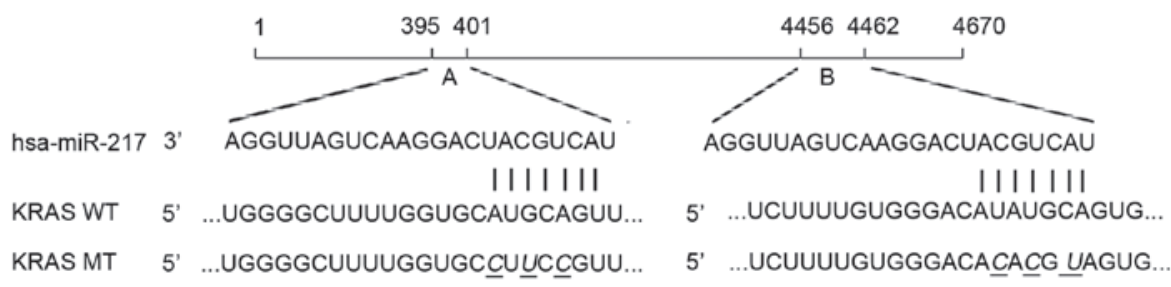

D

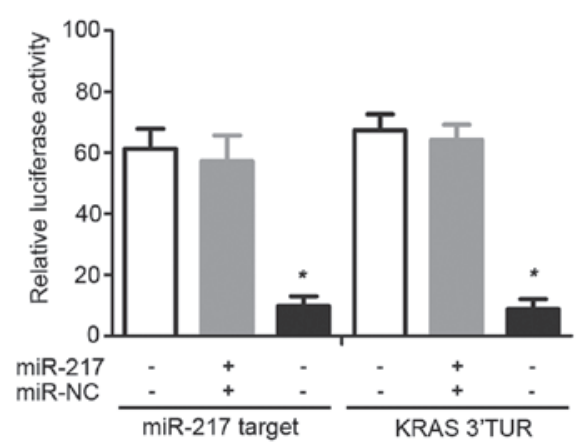

Figure 4. (A) KRAS and Raf-1 mRNA expression was measured by reverse transcription-quantitative polymerase chain reaction. "P $<0.05$ compared with the control groups. (B) Western blot analysis revealed reduced KRAS and Raf-1 protein levels in RKO cells transfected with miR-217 compared with miR-NC-transfected and mock-transfected RKO cells. (C) Schematic representation of the KRAS mRNA sequence. Predicted consequential pairing of KRAS region: Region A, positions 395-401 of KRAS 3' UTR; region B, positions 4456-4462 of KRAS 3' UTR. (D) RKO cells were co-transfected with luciferase reporter plasmids containing WT or MT miR-217 target sites in the KRAS 3' UTR or with miR-217 or miR-NC precursors. "P<0.01 compared with the miR-NC- and mock-transfected controls. mRNA, messenger RNA; NC, negative control; miR, microRNA; UTR, untranslated region; hsa, Homo sapiens; WT, wild type; MT, mutant.

progression, with a strong correlation between Bcl-2, Bcl-xl and miR-217 in CRC cells.

The MAPK family member MAPK1 regulates apoptosis and proliferation in cancer via the Bcl-2 and Bcl-xl apoptotic signaling pathways (24). To determine whether these pathways are involved in miR-217-induced RKO growth and inhibition of apoptosis, the present study first explored whether changing MAPK1 expression altered the miR-217-induced effects on apoptosis and growth. Second, the MAPK1, Bcl-2 and Bcl-xl expression levels in RKO cells overexpressing miR-217 were investigated.

To examine the function of miR-217-induced MAPK1 signaling in cell proliferation and apoptosis, RKO cells expressing miR-217 were stably transfected with full-length MAPK1. MTT assay (Fig. 3C), colony formation assay (Fig. 3D) and apoptosis assay (Fig. 3E) demonstrated that upregulation of MAPK1 in RKO cells overexpressing miR-217 reversed the increased apoptosis and suppressed the cell proliferation observed in RKO cells expressing only miR-217, strongly suggesting that MAPK1 is a critical downstream of miR-217 signaling. In addition, it was noticed that MAPK1,
$\mathrm{Bcl}-\mathrm{xl}$ and Bcl-2 expression were significantly lower in RKO cells transfected with miR-217 compared with RKO cells transfected with miR-NC (Figs. 1I, 3A and 3B), suggesting that miR-217 is an important regulator of the MAPK1-induced apoptotic pathway. Furthermore, upregulation of MAPK1 by expression of full-length MAPK1 substantially increased Bcl-2 and Bcl-xl expression in RKO cells stably expressing miR-217, compared with cells expressing only miR-217 (Fig. 3F). These results suggest that MAPK1, Bcl-xl and Bcl-2 are important downstream effectors of miR-217-induced inhibition of apoptosis and enhanced proliferation in CRC cells.

miR-217 regulates the RAS-MAPK signaling pathway. The present study further explored the effects of miR-217 overexpression in the MAPK signaling pathway. It was observed that the mRNA levels of KRAS and Raf-1, significant upstream components of the MAPK pathway $(25,26)$, were decreased by 0.32 - and 0.45 -fold, respectively, in miR-217-transfected RKO cells by using RT-qPCR (Fig. 4A). Western blotting revealed similar results at the protein level (Fig. 4B). These findings indicated that miR-217 may be a critical regulator of 
the RAS-Raf-MAPK pathway. Since miRNAs could regulate one pathway by affecting its multiple and functionally related targets (10), the current study explored whether miR-217 could modulate KRAS and Raf-1. It was revealed that there are no miR-217 target sites in the Raf-1 3' UTR, but two putative sites (A and B) were identified in the KRAS 3' UTR (Fig. 4C). These two target sites significantly inhibited the luciferase activity, which is consistent with a previous study reporting that miR-217 targets the oncogene KRAS in pancreatic ductal adenocarcinoma cells (15) (Fig. 4D). These data suggest that miR-217 can modulate KRAS and MAPK1 expression via directly binding to its target sites, indicating that miR-217 can inhibit RKO cell proliferation and enhance apoptosis by regulating the RAS/Raf/MAPK signaling pathway.

\section{Discussion}

miR-217, a novel of regulator of gene expression, is a special miRNA that can act as an oncogene or as a tumor suppressor gene depending on the cell type (27). Previous studies have shown that miR-217 acts as a tumor suppressor by targeting the oncogene SirT1 in endothelial cells (13), while it also acts as an oncogene by targeting PTEN, a tumor suppressor gene, in kidney cells and by enhancing the germinal center (GC) reaction in GC B-cells $(16,28)$. These findings prompted us to explore its expression and biological function in CRC. The current study determined the miR-217 expression levels in clinical CRC specimens and revealed that miR-217 expression was significantly associated with CRC histopathology. KaplanMeier analysis indicated that patients with CRC expressing high miR-217 levels had significantly longer overall survival than patients with low miR-217 expression, suggesting that miR-217 may play a critical role as a negative regulator of CRC development and progression. Furthermore, statistical analyses demonstrated that the miR-217 expression level was an independent prognostic parameter of patient outcomes. Thus, this is the first study to show that miR-217 expression is a novel molecular marker for predicting the prognosis of CRC.

The present study demonstrated that miR-217 suppresses tumor growth and enhances apoptosis in RKO CRC cells by blocking a novel miR-217 target, MAPK1. This protein has been regarded as an integration point for multiple biochemical signals, and is associated with various cellular processes, including proliferation, apoptosis, transcription regulation and development (29). Furthermore, it was demonstrated that upregulation of miR-217 markedly increased the apoptosis of RKO cells. Western blot assay demonstrated that RKO cells overexpressing miR-217 could decrease the protein expression levels of Bcl-2 and Bcl-xl, which are important molecules of the cellular apoptosis pathway $(30,31)$.

The current results revealed a novel mechanism by which miR-217 suppresses cell growth and enhances apoptosis in RKO cells. miR-217 mediates MAPK1, Bcl-2 and Bcl-xl expression, which play critical roles in CRC cell apoptosis $(32,33)$. Importantly, upregulation of miR-217 in RKO cells enhanced apoptosis and inhibited growth, an effect that was significantly reversed by constitutive MAPK1 expression. Furthermore, MAPK1 activation resulted in a significant increase in Bcl-2 and Bcl-xl expression in RKO cells stably expressing miR-217 compared with cells expressing only miR-217. MAPK1 is a key regulatory component of the intrinsic apoptotic pathway that is required for programmed cell death (34). The intrinsic pathway of apoptosis regulates the activity of the Bcl-2 family proteins (Bcl-2 and $\mathrm{Bcl}-\mathrm{xl})$ that control the integrity of the mitochondrial membrane (35). Our results are consistent with previous studies reporting that MAPK could suppress the activation of apoptosis by increasing the level of Bcl-2 and Bcl-xl $(36,37)$. The above finding demonstrates that miR-217 is required for RKO proliferation and apoptosis via MAPK1-induced intrinsic apoptosis signaling.

The present study also explored the function of miR-217 in the MAPK pathway to clarify the critical mechanism underlying tumorigenesis, proliferation and apoptosis. The MAPK pathway is regarded as an important cascade of proteins in the cell, transferring signals to the nucleus from receptors on the surface of the cell (38). This is a critical step for the progression of tumors $(39,40)$. Activated RAS phosphorylates and activates MAPK, and then produces certain changes in the cell, such as cell proliferation, apoptosis, invasion and drug resistance $(41,42)$. It was observed that KRAS, which is an important upstream signal of the MAPK pathway $(43,44)$, also contained miR-217 target sites in the 3' UTR. Since the transcription of KRAS, Raf-1 and MAPK1, critical members of the MAPK pathway $(25,43)$, was significantly reduced in RKO cells treated with miR-217, it was concluded that miR-217 modulates the RAS/Raf/MAPK signaling pathway. The RAS/Raf/MAPK pathway is well documented to affect the cellular apoptotic pathway, which is characterized by promoting cytochrome $c$ release (25). RAS/MAPK activity has been correlated with the downregulation of antiapoptotic components such as Bcl-2 and Bcl-xl (25). Indeed, the MAPK-activated Bcl-2 protein family has been shown to directly affect the release of cytochrome $c$ from mitochondria, offering a possible explanation for the observed cell apoptosis alteration (25).

In conclusion, the present study provides the first line of evidence that miR-217 suppresses tumor growth and enhances apoptosis in CRC, and that these effects are associated with the downregulation of RAS/Raf/MAPK signaling. Our results suggest that miR-217 is a promising therapeutic target for the treatment of CRC.

\section{References}

1. Debarros M and Steele SR: Colorectal cancer screening in an equal access healthcare system. J Cancer 4: 270-280, 2013.

2. Cunningham D, Atkin W, Lenz HJ, Lynch HT, Minsky B, Nordlinger B and Starling N: Colorectal cancer. Lancet 375: 1030-1047, 2010

3. Qaseem A, Denberg TD, Hopkins RH Jr, Humphrey LL, Levine J, Sweet DE and Shekelle P; Clinical Guidelines Committee of the American College of Physicians: Screening for colorectal cancer: A guidance statement from the American College of Physicians. Ann Intern Med 156: 378-386, 2012

4. Ragnhammar P, Hafström L, Nygren P and Glimelius B; SBU-group. Swedish Council of Technology Assessment in Health Care: A systematic overview of chemotherapy effects in colorectal cancer. Acta Oncol 40: 282-308, 2001.

5. Bergsland EK: Is more not better? Combination therapies in colorectal cancer treatment. Hematol Oncol Clin North Am 29: 85-116, 2015.

6. Saltz LB and Minsky B: Adjuvant therapy of cancers of the colon and rectum. Surg Clin North Am 82: 1035-1058, 2002.

7. Bauer TW and Spitz FR: Adjuvant and neoadjuvant chemoradiation therapy for primary colorectal cancer. Surg Oncol 7: 175-181, 1998.

8. Moss JA: Gene therapy review. Radiol Technol 86: 155-184, 2014. 
9. Roth JA and Cristiano RJ: Gene therapy for cancer: What have we done and where are we going? J Natl Cancer Inst 89: 21-39, 1997.

10. Kalla R, Ventham NT, Kennedy NA, Quintana JF, Nimmo ER, Buck AH and Satsangi J: MicroRNAs: New players in IBD. Gut 64: 504-517, 2015.

11. Xue J, Niu J, Wu J and Wu ZH: MicroRNAs in cancer therapeutic response: Friend and foe. World J Clin Oncol 5: 730-743, 2014.

12. Kong YW, Ferland-McCollough D, Jackson TJ and Bushell M: MicroRNAs in cancer management. Lancet Oncol 13: e249-e258, 2012.

13. Menghini R, Casagrande V, Cardellini M, MartelliE, Terrinoni A, Amati F, Vasa-Nicotera M, Ippoliti A, Novelli G, Melino G, et al: MicroRNA 217 modulates endothelial cell senescence via silent information regulator 1. Circulation 120: 1524-1532, 2009.

14. Xia H, Ooi LL and Hui KM: MicroRNA-216a/217-induced epithelial-mesenchymal transition targets PTEN and SMAD7 to promote drug resistance and recurrence of liver cancer. Hepatology 58: 629-641, 2013.

15. Zhao WG, Yu SN, Lu ZH, Ma YH, Gu YM and Chen J: The miR-217 microRNA functions as a potential tumor suppressor in pancreatic ductal adenocarcinoma by targeting KRAS Carcinogenesis 31: 1726-1733, 2010.

16. Kato M, Putta S, Wang M, Yuan H, Lanting L, Nair I, Gunn A, Nakagawa Y, Shimano H, Todorov I, et al: TGF-beta activates Akt kinase through a microRNA-dependent amplifying circuit targeting PTEN. Nat Cell Biol 11: 881-889, 2009.

17. Voong LN, Slater AR, Kratovac S and Cressman DE: Mitogen-activated protein kinase ERK1/2 regulates the class II transactivator. J Biol Chem 283: 9031-9039, 2008.

18. Giehl K, Skripczynski B, Mansard A, Menke A and Gierschik P Growth factor-dependent activation of the Ras-Raf-MEK-MAPK pathway in the human pancreatic carcinoma cell line PANC-1 carrying activated K-ras: Implications for cell proliferation and cell migration. Oncogene 19: 2930-2942, 2000

19. Lu Y, Xiao T, Zhang F, Chen Y, Liu Y, Li Y, Chen YD, Li Z and Guan M: Effect of mitochondrial tRNA(Lys) mutation on the clinical and biochemical characteristics of Chinese essential hypertensive subjects. Biochem Biophys Res Commun 454: 500-504, 2014

20. Wang Q, Qian J, Wang J, Luo C, Chen J, Hu G and Lu Y: Knockdown of RLIP76 expression by RNA interference inhibits invasion, induces cell cycle arrest, and increases chemosensitivity to the anticancer drug temozolomide in glioma cells. J Neurooncol 112: 73-82, 2013.

21. Hui AB, Shi W, Boutros PC, Miller N, Pintilie M, Fyles T, McCready D, Wong D, Gerster K, Waldron L, Jurisica I, et al Robust global micro-RNA profiling with formalin-fixed paraffinembedded breast cancer tissues. Lab Invest 89: 597-606, 2009.

22. Livak KJ and Schmittgen TD: Analysis of relative gene expression data using real-time quantitative PCR and the $2^{-\Delta \Delta C T}$ method. Methods 25: 402-408, 2001.

23. Wang Q, Wang JY, Zhang XP, Lv ZW, Fu D, Lu YC, Hu GH, Luo C and Chen JX: RLIP76 is overexpressed in human glioblastomas and is required for proliferation, tumorigenesis and suppression of apoptosis. Carcinogenesis 34: 916-926, 2013.

24. Subramanian M and Shaha C: Upregulation of Bcl-2 through ERK phosphorylation is associated with human macrophage survival in an estrogen microenvironment. J Immunol 179 : 2330-2338, 2007.

25. Vitagliano $\mathrm{O}$, Addeo R, D'Angelo $\mathrm{V}$, Indolfi $\mathrm{C}$, Indolfi $\mathrm{P}$ and Casale F: The Bcl-2/Bax and Ras/Raf/MEK/ERK signaling pathways: Implications in pediatric leukemia pathogenesis and new prospects for therapeutic approaches. Expert Rev Hematol 6: 587-597, 2013
26. Lemieux E, Cagnol S, Beaudry K, Carrier J and Rivard N: Oncogenic KRAS signalling promotes the Wnt/ $\beta$-catenin pathway through LRP6 in colorectal cancer. Oncogene 34: 4914-4927, 2015

27. Guo J, Feng Z, Huang Z, Wang H and Lu W: MicroRNA-217 functions as a tumor suppressor gene and correlates with cell resistance to cisplatin in lung cancer. Mol Cells 37: 664-671, 2014.

28. de Yébenes VG, Bartolomé-Izquierdo N, Nogales-Cadenas R, Pérez-Durán P, Mur SM, Martínez N, Di Lisio L, Robbiani DF, Pascual-Montano A, Cañamero M, et al: MiR-217 is an oncogene that enhances the germinal center reaction. Blood 124: 229-239, 2014

29. Joshi S and Platanias LC: Mnk kinase pathway: Cellular functions and biological outcomes. World J Biol Chem 5: 321-333, 2014.

30. Bates DJ and Lewis LD: Manipulating the apoptotic pathway: Potential therapeutics for cancer patients. Br J Clin Pharmacol 76: 381-395, 2013.

31. Zhang T and Saghatelian A: Emerging roles of lipids in BCL-2 family-regulated apoptosis. Biochim Biophys Acta 1831: 1542-1554, 2013

32. Ye L, Yuan G, Xu F, Sun Y, Chen Z, Chen M, Li T, Sun P, Li S and Sun J: The small-molecule compound BM-1197 inhibits the antiapoptotic regulators Bcl-2/Bcl-xL and triggers apoptotic cell death in human colorectal cancer cells. Tumour Biol 36: 3447-3455, 2015

33. Zhao SL, Hong J, Xie ZQ, Tang JT, Su WY, Du W, Chen YX, Lu R, Sun DF and Fang JY: TRAPPC4-ERK2 interaction activates ERK1/2, modulates its nuclear localization and regulates proliferation and apoptosis of colorectal cancer cells. PLoS One 6: e23262, 2011

34. Abdel-Magid AF: ERK2 inhibitors may provide treatment for cancer. ACS Med Chem Lett 4: 576-577, 2013.

35. Adams JM and Cory S: The Bcl-2 protein family: Arbiters of cell survival. Science 281: 1322-1326, 1998.

36. Wang D, Weng Q, Zhang L, He Q and Yang B: VEGF and Bcl-2 interact via MAPKs signaling pathway in the response to hypoxia in neuroblastoma. Cell Mol Neurobiol 29: 391-401, 2009.

37. Scheid MP, Schubert KM and Duronio V: Regulation of bad phosphorylation and association with $\mathrm{Bcl}-\mathrm{x}(\mathrm{L})$ by the MAPK/ Erk kinase. J Biol Chem 274: 31108-31113, 1999.

38. Orton RJ, Sturm OE, Vyshemirsky V, Calder M, Gilbert DR and Kolch W: Computational modelling of the receptortyrosine-kinase-activated MAPK pathway. Biochem J 392: 249-261, 2005.

39. Cheng Y, Zhang G and Li G: Targeting MAPK pathway in melanoma therapy. Cancer Metastasis Rev 32: 567-584, 2013.

40. Furukawa K and Hohmann S: Synthetic biology: Lessons from engineering yeast MAPK signalling pathways. Mol Microbiol 88: 5-19, 2013.

41. Pritchard AL and Hayward NK: Molecular pathways: Mitogen-activated protein kinase pathway mutations and drug resistance. Clin Cancer Res 19: 2301-2309, 2013.

42. Zassadowski F, Rochette-Egly C, Chomienne C and Cassinat B: Regulation of the transcriptional activity of nuclear receptors by the MEK/ERK1/2 pathway. Cell Signal 24: 2369-2377, 2012.

43. Collins MA and Pasca di Magliano M: Kras as a key oncogene and therapeutic target in pancreatic cancer. Front Physiol 4: 407, 2013.

44. Sundaram MV: RTK/Ras/MAPK signaling. WormBook: 1-19, 2006. 